\title{
Plano de Reorganização da Atenção à Hipertensão Arterial e ao Diabetes Mellitus: fase de detecção de casos suspeitos de DM
}

\author{
Brazillian national strategy for the Reorganization of Care for Arterial \\ Hipertension and Diabetes Mellitus: the experience of diabettes mass screning
}

Secretaria de Políticas Públicas/MS*

O Plano de Reorganização da Atenção à Hipertensão Arterial e ao Diabetes Mellitus tem como principal objetivo estabelecer diretrizes e metas para a atenção aos portadores desses agravos no Sistema Único de Saúde, mediante a reestruturação e a ampliação do atendimento básico voltado para a hipertensão arterial e o diabetes mellitus, com ênfase na prevenção primária, na ampliação do diagnóstico precoce e na vinculação de portadores à rede básica de saúde.

As doenças do aparelho circulatório e o diabetes representam importantes problemas de saúde pública no País. Há algumas décadas, as doenças cardiovasculares são a primeira causa de morte no Brasil, segundo os registros oficiais (Sistema de Informação sobre Mortalidade-SIM). Em 2000, corresponderam a mais de $27 \%$ do total de óbitos, ou seja, neste ano, 255.585 pessoas morreram em conseqüência de doenças do aparelho circulatório.

A hipertensão arterial sistêmica (HAS) e o diabetes mellitus (DM) constituem os principais fatores de risco para as doenças do aparelho circulatório. A HA está relacionada a $80 \%$ dos casos de acidente vascular encefálico (AVE) e a $60 \%$ dos casos de doença isquêmica do coração. Entre as complicações mais freqüentes decorrentes do DM, encontram-se o infarto agudo do miocárdio (IAM), o AVE, a insuficiência renal crônica, as amputações de pés e pernas, a cegueira definitiva, os abortos e as mortes perinatais.

O número de internações por DM registra- do no Sistema de Informação Hospitalar (SIH/ SUS) é elevado, tendo sido gastos mais de R\$ 39 milhões com hospitalizações no SUS em 2000. Esses custos estão relacionados à alta taxa de permanência hospitalar do diabético e também à severidade das complicações que, muitas vezes, demandam procedimentos de alta complexidade.

Os dados do estudo multicêntrico de diabetes no Brasil realizado em 1987 pelo Ministério da Saúde, Sociedade Brasileira de Diabetes e CNPq demonstraram uma prevalência de 7,6 \% na população de 30 a 69 anos de idade. O estudo revelou um alto grau de desconhecimento em relação à doença (mais de 50\% dos diagnosticados não sabiam ser portadores de diabetes).

Nos pacientes que conheciam o seu diagnóstico, uma análise do tipo de tratamento revelou que $23 \%$ não faziam nenhum tipo de tratamento, demonstrando a inadequação do acompanhamento dessa patologia.

Estudos randomizados, tanto em diabetes tipo 1 quanto em diabetes tipo 2, demonstraram claramente a redução das complicações crônicas com o bom controle metabólico da doença.

A identificação precoce dos casos e o estabelecimento do vínculo entre os portadores e as Unidades Básicas de Saúde são elementos imprescindíveis para o sucesso do controle desses agravos. O acompanhamento e o controle da HA e do DM, no âmbito da atenção básica, evitam o agra- 
vamento dessas patologias e o surgimento de complicações, reduzindo o número de internações hospitalares, bem como a mortalidade por doenças cardiovasculares.

Apesar da existência de várias experiências municipais bem-sucedidas quanto à garantia do acompanhamento dos casos de HA e DM, em grande parte do País observa-se falta de vínculo entre os portadores e as unidades de saúde. Em geral, o atendimento aos pacientes ocorre de modo assistemático nos serviços de emergência, sem a garantia da identificação de le-

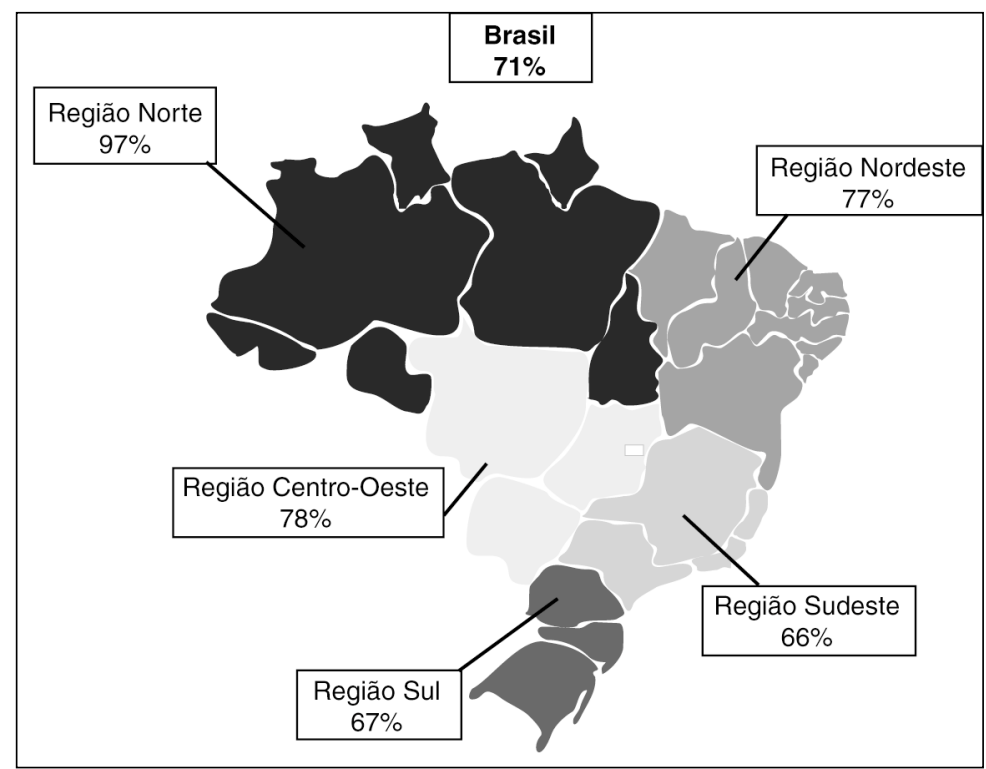

Figura 1 - Desempenho nacional da campanha, percentagem da população que realizou o exame de detecção durante a campanha por regiōes. Brasil, março, 2001. sões em órgãos-alvo e do tratamento e controle adequados a cada caso. No campo da promoção da saúde e da prevenção dos fatores de risco, a situação é ainda mais crítica, tendo em conta a falta de tradição dos serviços de saúde na realização sistemática de tais ações.

O Ministério da Saúde, com o propósito de contribuir para a redução da morbimortalidade associada a HA e ao DM, assumiu o compromisso de estabelecer uma parceria com Estados, municípios e sociedade para apoiar a reorganização da rede de saúde, com vistas à melhoria da atenção aos portadores de diabetes, mediante o desenvolvimento de ações articuladas de promoção, prevenção, tratamento e recuperação.

\section{A campanha e seus resultados}

A Campanha Nacional de Detecção de Casos Suspeitos de Diabetes, que, entre outras ações, integra o Plano de Reorganização da Atenção à Hipertensão Arterial e ao Diabetes Mellitus, aconteceu nas 33.000 Unidades Básicas de Saúde (UBS) nos meses de março e abril deste ano, realizando $20,7 \mathrm{mi}$ lhões de glicemias capilares. Identificaramse 2,9 milhões de suspeitos de diabetes, sendo que, aproximadamente, um milhão dessas pessoas é hipertensa e portadora de diabetes. Uma ação desse porte, além do bene- fício de detectar novos casos, contribui para a estruturação do atendimento aos portadores de diabetes na rede básica.

É o primeiro screening para detecção de casos suspeitos de diabetes mellitus no Brasil uma iniciativa pioneira em termos mundiais -, uma vez que não existe, na literatura, relato de nenhuma experiência nacional, com testagem em massa da população, utilizando o sistema público de saúde (Figura 1).

Com relação ao desempenho das cinco regiões brasileiras, apesar das dificuldades históricas, a região Norte teve o melhor desempenho, conseguindo testar cerca de $97 \%$ de sua população-meta. As regiões Nordeste e Centro-Oeste obtiveram um resultado semelhante, conseguindo testar, respectivamente, $77 \%$ e $78 \%$ de sua população. As regiões Sul e Sudeste tiveram cobertura de $67 \%$ e $6 \%$, respectivamente (Figura 2).

Do total de exames realizados, 14,66\% apresentaram resultado anormal, representando 2,9 milhões de exames suspeitos de diabetes mellitus. A prevalência do diabetes mellitus na população será calculada mediante estudo nacional de avaliação do impacto da Campanha. O alto número de testes positivos é compatível com o que é de se esperar de um teste altamente sensível como a glicemia capilar. 


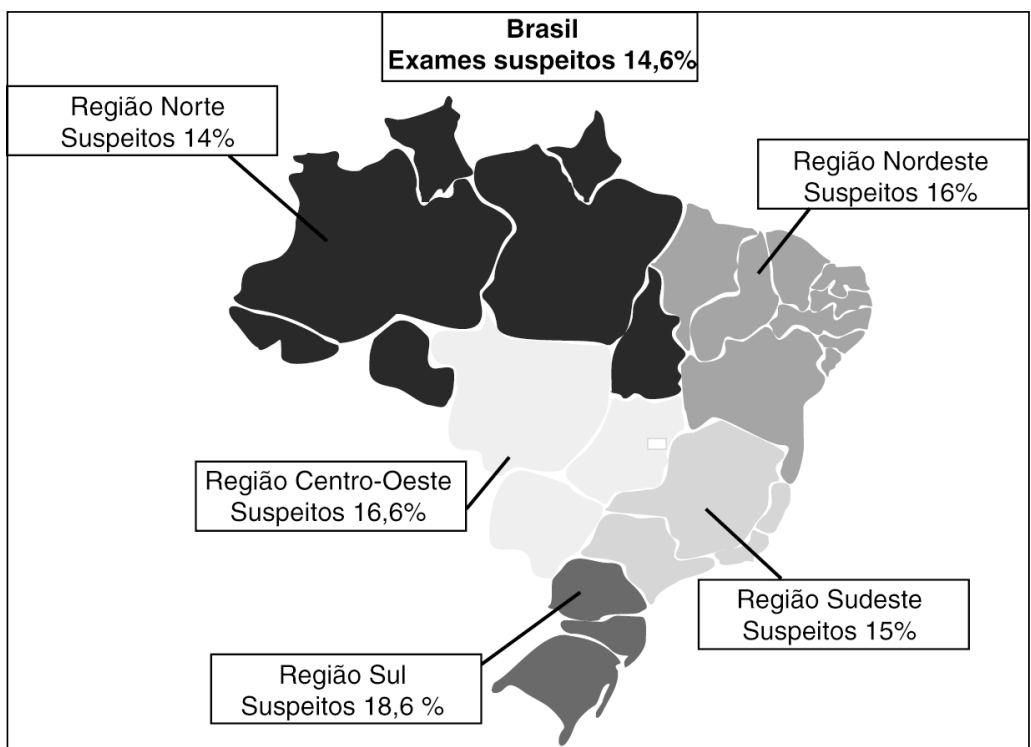

Figura 2 - Exames detectados como suspeitos por regiões. Brasil, março, 2001.
$80 \%$. Observou-se uma baixa cobertura em apenas $2 \%$ dos municípios em que a população cadastrada no PSF era superior a $50 \%$.

Em contrapartida, foi observada baixa cobertura em $8 \%$ do conjunto de municípios sem PSF ou naqueles municípios em que a população cadastrada no Programa era inferior a $50 \%$.

O mesmo foi observado quando se avaliou a cobertura da Campanha em relação ao Programa de Agentes Comunitários (PACS).

O resultado desse teste deve ser confirmado com a glicemia plasmática, ou seja: o paciente que obteve um resultado alterado deverá realizar uma consulta no posto de saúde para confirmar, ou não, o diagnóstico (Figura 3).

Nas 27 capitais, foram realizados 4.541 .999 exames, correspondendo a 60\% (7.607.766) da meta esperada nesses municípios. No grupo foram identificados 699.265 casos suspeitos para o diabetes mellitus, $15 \%$ do total da população testada.

Análises preliminares dos dados disponíveis no Sistema de Informação Ambulatorial mostram que a cobertura da Campanha (número de exames realizados em relação à meta, considerando a meta como $75 \%$ da população com mais de 40 anos de idade), foi maior naqueles municípios com alto índice de cobertura do Programa de Saúde Família (PSF) e naqueles habilitados em algum nível de gestão pela Norma Operacional Básica (NOB/96).

Para fins de análise estatística, definiu-se como baixa uma cobertura menor que $50 \%$ e como alta uma cobertura maior que
Outras características dos municípios influenciaram a cobertura da Campanha. Nos municípios com mais de $75 \%$ da população em área urbana, com maior índice de alfabetização e escolaridade, foi observada uma menor cobertura da Campanha, comparados aos municípios com maior proporção de população rural e com menor índice de alfabetização e escolaridade. A cobertura da Campanha em municípios com mais de 100 mil habitantes e municípios das regiões metropolitanas ficou em torno de $50 \%$, enquanto nos municípios com menos de 100 mil habitantes, a cobertura foi de $81 \%$ e, fora das áreas metropolitanas, de $75 \%$.

Em conclusão, a implementação das dire-

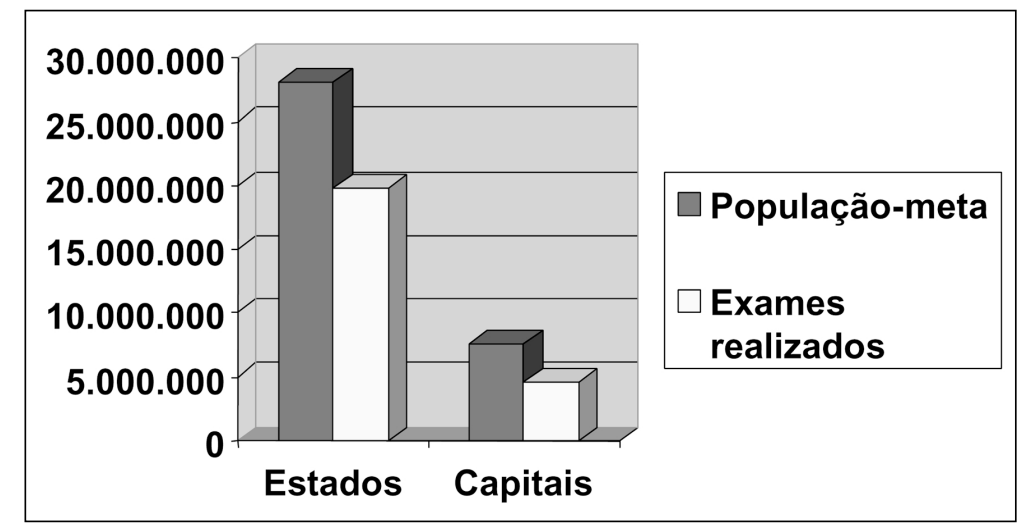

Figura 3 - Desempenho das principais capitais. População-meta em comparação ao número de exames realizados nos principais Estados e capitais. Brasil, março, 2001. 
trizes do Plano de Reorganização da Atenção à Hipertensão e ao Diabetes Mellitus representa um dos grandes desafios para o Sistema Único de Saúde.

Mesmo considerando os avanços importantes na organização da atenção básica, especialmente quanto à ampliação do financiamento e à expansão dos serviços nesse nível de atenção, constatam-se importantes pontos de estrangulamento quanto à capacidade dessa rede de agir ativamente, baseada em critérios de risco, promovendo a busca ativa e a vinculação dos usuários às equipes assistenciais.

Esse novo papel para a rede básica também se coloca em relação a várias ações de saúde, tais como na assistência pré-natal - captação precoce de mulheres no primeiro trimestre; na eliminação da hanseníase e no controle da tuberculose; na ampliação das vacinações obrigatórias à população rural e à periferia dos grandes centros urbanos etc.

Entretanto, entre esses desafios, um dos maiores é, certamente, a mudança na forma de abordar as doenças não transmissíveis, especialmente a hipertensão e o diabetes. Para essas doenças, pelas suas características patológicas e seus determinantes, não bastam a detecção e a identificação dos portadores. Mais do que isso, é preciso preparar as unidades e suas equipes para dar seguimento, acolher, transferir informações, tratar de maneira conveniente, referenciar e avaliar os impactos sobre as condições clínicas dos usuários ou mesmo sobre o conjunto da comunidade.

Esse é, sem dúvida, o grande desafio do Plano de Reorganização da Atenção à Hipertensão Arterial e ao Diabetes Mellitus, com os seus vários componentes.

Esse é o principal produto, em médio e longo prazos, da Campanha Nacional de Detecção de Casos Suspeitos: realizar uma campanha pensando nos aspectos estruturais que se colocam para a atenção básica no País, ser porta de entrada resolutiva, acolhedora e reorganizadora do processo mais complexo que é a atenção individual e coletiva dos usuários do SUS. 